\title{
Association of Plasma Complement System With Brain Structure Deficits in Bipolar and Major Depressive Disorders
}

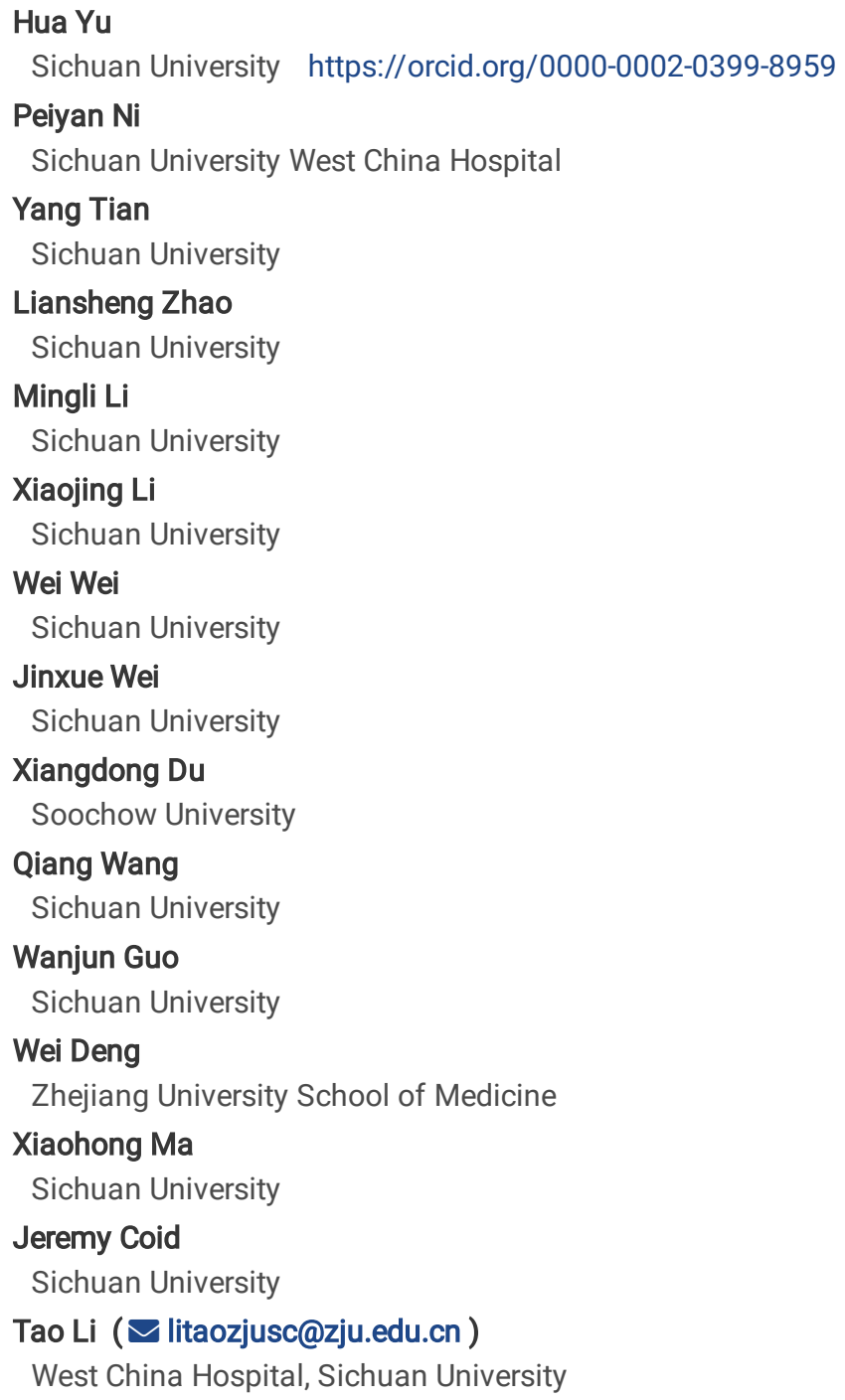

Research

Keywords: Bipolar disorder, major depressive disorder, inflammation, complement component, brain structure.

Posted Date: October 12th, 2021

DOI: https://doi.org/10.21203/rs.3.rs-922528/v1

License: (c) (i) This work is licensed under a Creative Commons Attribution 4.0 International License. Read Full License 


\section{Abstract}

Objective Inflammation plays a crucial role in the pathogenesis of major depressive disorder (MDD) and bipolar disorder (BD). However, the underlying neurobiological mechanisms are poorly understood. This study aimed to examine whether the dysregulation of complement components contributes to brain structure deficits in BD and MDD patients.

Methods A total of $52 \mathrm{BD}$ patients, $35 \mathrm{MDD}$ patients, and 53 mentally healthy controls were recruited from the inpatient and outpatient departments of West China Hospital of Sichuan University. The human complement panel 2-immunology multiplex assay was used to measure the levels of complement $\mathrm{C} 1 \mathrm{q}, \mathrm{C} 3, \mathrm{C} 3 \mathrm{~b}, \mathrm{C} 4$, factor $\mathrm{B}$, factor $\mathrm{H}$, and properdin. Whole brain-based comparison was performed to investigate differences in gray matter volume and cortical thickness among the BD, MDD, and control groups, and relationships were explored between neuroanatomical differences and levels of complement components.

Results The gray matter volume in the medial orbital frontal cortex (MOFC) and middle cingulum decreased in both patient groups, while the cortical thickness of the left precentral and left superior frontal gyrus was affected differently. $\log _{10}$-transformed concentrations of $\mathrm{C} 1 \mathrm{q}, \mathrm{C} 4$, factor $\mathrm{B}$, factor $\mathrm{H}$, and properdin were higher in both patient groups than in controls, while levels of $\mathrm{C} 1 \mathrm{q}$, factor $\mathrm{H}$, and properdin showed a significant negative correlation with gray matter volume in the MOFC at the voxel-wise level.

Conclusion Greater inflammation in mOFC was observed in BD and MDD patients than in controls. Structural deficits in both patient groups were associated with elevated levels of certain complement factors, providing insight into the neuro-inflammatory pathogenesis of mood disorders.

\section{Introduction}

Major depressive disorder (MDD) and bipolar disorder (BD) are severe psychiatric diseases with overlapping symptomatology [1]. Despite the high global lifetime prevalence of MDD (13.2\%) [2] and bipolar spectrum disorder (2.4\%) [3], the underlying pathophysiology is poorly understood [1]. BD is characterized mainly by recurrent mood exacerbations of opposite polarity, ranging from major depressive to manic episodes [4], with depression being the most common presentation [5]. However, patients with BD are often misdiagnosed with MDD for many years, leading to inadequate treatment, poor prognosis, high healthcare costs, and serious adverse events such as mania or increased suicidality [6]. Therefore, there is an increasing need to identify biomarkers that could detect the differences in pathophysiology between MDD and $\mathrm{BD}$ and help improve treatment response $[7,8]$.

Brain structural abnormalities have recently been identified as promising candidate biomarkers for affective disorders, improving their prevention and treatment [9]. Since changes in gray matter volume (GMV) and cortical thickness (CT) can reflect degeneration, loss of neuroplasticity, or neurotoxicity [10], volume-based grey matter measures, such as voxel-based morphometry (VBM), and CT-based measures, such as surface-based morphometry (SBM), are widely used for brain structure analysis [11]. In fact, GMV and CT alterations have been identified as significant biomarkers of first-episode BD and MDD $[1,12,13]$. For instance, GMV in the medial orbital frontal cortex (mOFC) was reduced to a similar extent in BD and MDD patients in previous work $[13,14]$. In a VBM-based study, both MDD and BD patients showed smaller GMV in the left anterior cingulate cortex (ACC) and right hippocampus than healthy controls did, while GMV in the left superior frontal gyrus (SFG) and left ACC was lower in MDD than in BD patients [15]. In another study, BD patients showed significantly smaller CT in the frontal lobe than controls or patients with MDD, while both patient groups showed significantly smaller CT in the left inferior temporal cortex than controls [1]. In contrast, another study found no CT differences between MDD and BD patients [16]. These findings suggest similarities and differences in GMV and CT alterations in mood disorders, yet the underlying neuropathology has not yet been elucidated.

Structural changes in the brain have been associated with stress, genetic factors, and environmental factors [17]. Inflammation has also been implicated in the neuropathology of mood disorders [18]. Inflammation involves cytokine cascades, cellular immune responses, increased levels of acute-phase proteins, and altered levels of complement components [19]. Elevated levels of peripheral pro-inflammatory mediators, such as pro-inflammatory cytokines and immune mediators in plasma, have been identified in BD and other mood disorders, while patients with systemic autoimmune diseases may be at higher risk of BD and MDD [18, 20].

The complement system is a set of immune proteins that is involved in first-line defense against pathogens and waste removal, and it mediates between innate and acquired immune responses [21]. There are three major pathways of complement activation: classical, mannanbinding lectin, and alternative [22]. All pathways converge on the generation of C3 convertase, which cleaves complement C3 into C3a and C3b [22]. C1q is the primary initiator of the classical pathway and plays an important recognition role in adaptive and innate immunity [23]. The complement components $\mathrm{C} 1 \mathrm{q}$, iC3b, C3, and $\mathrm{C} 4$ are involved in classical pathways, while properdin, factor $\mathrm{B}$, factor $\mathrm{H}$, and iC $3 \mathrm{~b}$ are involved in alternative pathways [24]. MDD patients show increased serum levels of C1q, C3, C4, and factor $\mathrm{H}$ [25-27]. However, inconsistent results have been reported for BD: different studies have reported increased or decreased C3 levels [28, 29]. BD patients are known to present more severe 
dysregulation of interleukin 6 (IL-6), tumor necrosis factor-a, and C-reactive protein than MDD patients [30, 31], but whether the two types of patients also differ in plasma levels of complement factors is unclear.

Rodent and human brain imaging studies have shown that several cortical and subcortical structures in BD and MDD patients, including the amygdala, striatum, insula, ACC, orbitofrontal cortex, and hippocampus/parahippocampus, are particularly sensitive to changes in peripheral inflammation, especially to elevated levels of C-reactive protein and cytokines [32-34]. However, only a few studies have examined the association between complement components and brain structure in mood disorders. Significantly increased C3 expression was found in the post-mortem prefrontal cortex of depressed suicide subjects as well as in mice with chronic stress-induced depressive-like behavior [26]. In another study, genetic variants of $C 4$, which encodes a protein of the classical pathway, have been associated with schizophrenia development, providing a solid basis for establishing a causal relationship between complement-mediated synaptic pruning and cortical thinning, which is frequently associated with psychiatric illness [35]. Animal studies have also consistently shown that activation of C1q, C4, and C3 contribute to synapse loss in Alzheimer's disease, leading to gray matter loss and brain atrophy [36]. In addition, complement components have been found to enhance pro-inflammatory signaling, leading to increased IL-1 $\beta$ production [26].

Despite previous attempts to determine the role of the complement system in the etiology of mental disorders, its effect on brain structure in BD and MDD remains unexplored. Since brain volume and CT can define endophenotypes for BD and MDD [37, 38], the relationship between inflammation and disturbed brain structure might provide insights into disease pathophysiology [39]. Therefore, in the present study, we evaluated the association of altered levels of complement components with brain structure deficits in patients with BD and MDD. We hypothesized that the two groups of patients would show common and distinct patterns of GMV and CT alterations, and that both groups would show increased levels of complement factors, especially the BD group. We also explored whether elevated complement factor levels in plasma, an indicator of inflammation, correlated with GMV or CT differences among BD patients, MDD patients and controls. Finally, we examined whether the observed differences in brain structure correlated with cognitive function.

\section{Methods}

\section{Participants}

Patients with BD and MDD were recruited from the inpatient and outpatient departments of West China Hospital of Sichuan University. BD or MDD was diagnosed using the structured clinical interview for DSM-IV Axis-I disorders (patient version) [40]. All subjects, with ages between 16-55 years, were right-handed Han Chinese who were physically and neurologically healthy. Their demographic characteristics were recorded, including disease duration, treatment history, illness stage, age, sex, education level, and body mass index (BMI). Manic and depressive symptoms were assessed using the Young Mania Rating Scale [41] and the Hamilton Rating Scale for Depression [42]. Patients with concurrent neurological illness, mental retardation, cardiovascular disease, schizophrenia, anxiety disorder, alcohol or drug abuse, and history of loss of consciousness were excluded from the study. None of the patients had received electroconvulsive therapy before participation.

For comparison, demographically matched healthy controls (HCs) were recruited from the local community through advertising. HCs were included if they (1) were 16-55 years old; (2) did not meet the diagnostic criteria of the structured clinical interview for DSM-I (non-patient edition) [43]; (3) had no current or past significant medical or physical disease, such as diabetes, thyroid diseases, hypercholesteremia, liver diseases, epilepsy, stroke, or systemic lupus erythematosus; and (4) had no history of psychiatric illness in first-degree relatives. All participants provided written informed consent after the study procedures were explained. The study was approved by the Institutional Review Board of Sichuan University.

\section{Cognitive function measurements}

Intelligence quotient (IQ), verbal IQ, and performance IQ scores of all participants were assessed using the seven-subtest short form (information, arithmetic, digital symbol, digital span test, block design, picture completion, and similarities) of the revised Wechsler Adult Intelligence Scale in Chinese [44]. The Logical Memory Test is a standard test used for assessing immediate and delayed recall memory function [45]. The Trail Making Test A/B [46] was used to measure attention, visual screening, and processing speed. The digital symbol substitution test was used to measure working memory, visuospatial processing, attention, and processing speed [44] (see the Supplementary Materials and the Methods section for more details).

\section{Measurement of complement component levels}

Blood samples were collected by venipuncture between 4.00 p.m. and 4.30 p.m. using ethylenediaminetetraacetic acid as an anti-coagulant. Peripheral blood mononuclear cells were removed by refrigerated centrifugation at 1,000 $\mathrm{g}$ for 10 min, and the separated plasma was immediately divided into $0.5-\mathrm{mL}$ aliquots and stored at $-80^{\circ} \mathrm{C}$. Plasma factors were evaluated using MILLIPLEX ${ }^{\circledR}$ MAP kits (Merck KGaA, Darmstadt, Germany). The human complement panel 2-immunology multiplex assay (HCMP2MAG-19K, Merck Millipore, Billerica, MA, USA) was used to measure the levels of $\mathrm{C} 1 \mathrm{q}, \mathrm{C} 3, \mathrm{C} 3 \mathrm{~b} / \mathrm{iC} 3 \mathrm{~b}, \mathrm{C} 4$, factor $\mathrm{B}$, properdin, and factor $\mathrm{H}$, following the manufacturer's instructions. Data were 
analyzed using a FLEXMAP 3D ${ }^{\circledR}$ instrument (Luminex, Merck Millipore, Billerica, MA, US) operated with xPONENT ${ }^{\circledR}$ software (version 4.0, Luminex). Standard curves were generated using standard samples in the multiplex assay kit. Median fluorescent intensity data were analyzed using a weighted 5-parameter logistic method to calculate the factor concentrations.

\section{Image acquisition}

Magnetic resonance imaging (MRI) was performed on a 3.0-T MR scanner (Achieva; Philips, Amsterdam, The Netherlands) using an eightchannel phased-array head coil. Foam padding and earplugs were used to minimize head movement and scanner noise. During scanning, participants were reminded to remain still with their eyes closed, without falling asleep or thinking of anything particular, which subjects confirmed immediately after MRI. High-resolution T1 images were acquired by 3D magnetization-prepared rapid gradient-echo sequence (repetition time, $8.37 \mathrm{~ms}$; echo time, $3.88 \mathrm{~ms}$; flip angle, $7^{\circ}$; in-plane matrix resolution, $256 \times 256$; field of view, $24 \times 24 \mathrm{~cm}{ }^{2}$; number of slices 188). During MRI, none of the participants showed more than $2 \mathrm{~mm}$ displacement in the $\mathrm{x}, \mathrm{y}$, or $\mathrm{z}$ direction or more than $2^{\circ}$ angular motion.

\section{VBM and SBM}

VBM and SBM analyses were conducted using the Computational Anatomy Toolbox 12 (CAT12, version 12.5, Jena University Hospital, Jena, Germany, http://dbm.neuro.uni-jena.de/cat/) [47], an extension of Statistical Parametric Mapping (University College London, London, UK, http://www.fil.ion.ucl.ac.uk/spm/software/spm12/), following the manufacturer's instructions (http://dbm.neuro.uni-jena.de/cat12/CAT12Manual.pdf). The T1 MRI scans of all subjects were registered to the Montreal Neurological Institute space. The whole-brain structural data were then segmented into white matter, gray matter, and cerebrospinal fluid, and bias was corrected to remove intensity non-uniformities. The images of segmented gray matter were used to assess the number of volume changes based on spatial registration, while the modulated images of gray matter could reflect the tissue volumes for VBM analysis. The total intracranial volume (TIV) of each subject was calculated and used as a covariate in further statistical analyses. The normalized gray matter images were smoothed using a Gaussian filter with a fullwidth half-maximum (FWHM) of $8 \mathrm{~mm}$. The CT of the left and right hemispheres was automatically estimated by CAT12 based on the projection-based thickness method, and the corresponding images were smoothed with a 15-mm FWHM Gaussian kernel [48].

\section{Statistical analysis}

Statistical analyses were carried out using SPSS 24.0 (IBM, Chicago, IL, USA). Differences in demographic and clinical data were assessed using one-way analysis of variance for continuous variables and Fisher's chi-squared test for categorical variables. Data normality was evaluated by visual inspection and the Kolmogorov-Smirnov test. The concentrations of all complement components were log $10^{- \text {transformed }}$ prior to analysis in order to normalize their distributions. Complement factors and cognitive function were compared using analysis of covariance (ANCOVA), while controlling for age, sex, education level, and BMI. Clinical parameters between the two patient groups were compared using the two-samples $t$ test. To explore correlations of complement components with cognitive function or other clinical parameters, partial correlation analysis was conducted after controlling for demographic parameters. Differences associated with $P<0.05$ were considered statistically significant.

\section{Image analysis}

Voxel-wise GMV and vertex-wise CT differences among the three subject groups were investigated using ANCOVA, while co-varying for age, sex, education level, and BMI. For GMV analysis, all voxels with GMV probability value $<0.1$ (absolute threshold; range, $0-1$ ) were excluded to avoid possible edge effects around the margin between different tissue types. TIV was also controlled as a covariate in GMV analysis. While controlling for age, sex, education level, and BMI, statistical maps were created to identify potential linear correlations between plasma levels of complement components and whole-brain GMV or CT in BD patients, MDD patients and HCs considered separately as three groups or together as one large group [49]. The threshold was set at $P<0.05$ after correcting for family-wise error rate at the cluster level with conservative voxelwise $P<0.0005$. To further analyze the relationship between GMV or CT and other variables, GMV and CT values were extracted, respectively, from the anatomical automatic labeling atlas or the Desikan-Killiany (DK40) cortical atlas, then subjected to partial correlation analysis using SPSS 24.0. The extracted values were used to identify regions that differed significantly between patients and controls or between different types of patients, as well as to identify neuroanatomical alterations that correlated significantly with levels of complement components.

\section{Results}

\section{Demographic and clinical characteristics of patients and HCs}

A total of 140 subjects, including 52 BD patients, 35 MDD patients, and 53 age- and sex-matched controls were enrolled in the study (Table 1). Across the three groups, there were no significant differences in sex, or smoking status. Moreover, there were no significant differences between BD and MDD patients in age at onset, total illness duration, number of depressive episodes, general assessment functioning, or anti-depressant usage. Both patient groups had a lower education level than HCs, while BD patients had a higher BMI than MDD patients and HCs. Although BD patients had lower Hamilton depression total scores than the MDD group, they were treated more often with mood stabilizers and/or

Page $4 / 17$ 
antipsychotics. Specifically, 24 of the 52 BD patients (46.15\%) received antidepressants, $30(57.69 \%)$ received mood stabilizers, and 31 (59.62\%) received antipsychotics. In contrast, 18 of the 35 patients with MDD (51.43\%) were taking antidepressants, while only 3 (8.57\%) received antipsychotics and none received mood stabilizers. 
Table 1

Demographic and clinical characteristics of BD, MDD and HC.

\begin{tabular}{|c|c|c|c|c|c|c|c|}
\hline & \multirow{2}{*}{$\begin{array}{l}\text { BD M (SD) } \\
(n=52)\end{array}$} & \multirow{2}{*}{$\begin{array}{l}\text { MDD M (SD) } \\
(n=35)\end{array}$} & \multirow{2}{*}{$\begin{array}{l}\text { HC M (SD) } \\
(n=53)\end{array}$} & \multicolumn{4}{|c|}{ ANOVA } \\
\hline & & & & $F / x^{2}$ & df & P, 2-tail & Post hoc test \\
\hline Age (Years) & $28.92(11.45)$ & $30.26(10.26)$ & $27.47(8.98)$ & 0.793 & 2 & 0.46 & - \\
\hline Education (years) & $13.12(3.23)$ & $12.49(4.15)$ & $15.15(3.60)$ & 6.86 & 2 & $0.001 * \star$ & $\mathrm{BD}, \mathrm{MDD}<\mathrm{HC}$ \\
\hline BMI & $23.10(3.86)$ & $21.10(2.95)$ & $21.33(3.23)$ & 4.78 & 2 & $0.010 *$ & $\mathrm{BD}>\mathrm{MDD}, \mathrm{HC}$ \\
\hline Gender (male /female) & $21(\mathrm{M}) / 31(\mathrm{~F})$ & $15(\mathrm{M}) / 20(\mathrm{~F})$ & $22(\mathrm{M}) / 31(\mathrm{~F})$ & 0.053 & 2 & 0.97 & - \\
\hline Smoking (yes/no) & 8 (yes)/38(no) & 7 (yes)/28 (no) & 5 (yes)/44 (no) & 1.73 & 2 & 0.422 & - \\
\hline \multicolumn{8}{|l|}{ Cognitive function } \\
\hline VIQ & $104.92(21.97)$ & $98.32(31.16)$ & $112.48(13.45)$ & 1.48 & 2 & 0.23 & - \\
\hline PIQ & $96.35(22.29)$ & $94.23(30.32)$ & 108.35 (13.05) & 1.90 & 2 & 0.15 & - \\
\hline WAIS IQ & $101.63(21.94)$ & $96.74(30.87)$ & $111.65(13.34)$ & 1.71 & 2 & 0.19 & - \\
\hline Logical memory immediately & $7.36(4.56)$ & $8.81(5.06)$ & $10.81(4.24)$ & 4.43 & 2 & $0.014 *$ & $\mathrm{BD}<\mathrm{HC}$ \\
\hline Logical memory delayed & $5.61(3.93)$ & $7.00(4.42)$ & $8.60(4.34)$ & 3.90 & 2 & $0.023 *$ & $\mathrm{BD}<\mathrm{HC}$ \\
\hline TMT-A-Time & $44.32(22.47)$ & $34.68(15.28)$ & $37.18(14.56)$ & 2.20 & 2 & 0.16 & - \\
\hline TMT-B-Time & 72.35 (39.29) & $61.83(45.68)$ & $56.00(39.32)$ & 1.28 & 2 & 0.28 & - \\
\hline DSST & $49.94(18.04)$ & $49.26(18.69)$ & $59.88(19.80)$ & 1.46 & 2 & 0.24 & - \\
\hline \multicolumn{8}{|l|}{ Complement component } \\
\hline $\log _{10} \mathrm{C1q}$ & $2.75(0.42)$ & $2.59(0.29)$ & $2.30(0.17)$ & 22.47 & 2 & $0.000 * * *$ & $\mathrm{BD}, \mathrm{MDD}>\mathrm{HC}$ \\
\hline $\log _{10} C 3$ & $2.24(0.35)$ & $2.00(0.24)$ & $1.97(0.23)$ & 10.30 & 2 & $0.000 * * *$ & $\mathrm{BD}>\mathrm{MDD}, \mathrm{HC}$ \\
\hline $\log _{10} C 4$ & $2.99(0.18)$ & $2.89(0.12)$ & $2.78(0.10)$ & 24.72 & 2 & $0.000 * * *$ & $\mathrm{BD}>\mathrm{MDD}>\mathrm{HC}$ \\
\hline $\log _{10}$ Factor B & $2.96(0.31)$ & $2.87(0.25)$ & $2.67(0.14)$ & 16.20 & 2 & $0.000 * * *$ & $\mathrm{BD}, \mathrm{MDD}>\mathrm{HC}$ \\
\hline $\log _{10}$ Factor H & $3.12(0.33)$ & $2.97(0.19)$ & $2.79(0.12)$ & 21.59 & 2 & $0.000 * * *$ & $\mathrm{BD}>\mathrm{MDD}>\mathrm{HC}$ \\
\hline $\log _{10}$ Properdin & $2.10(0.32)$ & $2.02(0.22)$ & $1.79(0.15)$ & 18.52 & 2 & $0.000 * * *$ & $\mathrm{BD}, \mathrm{MDD}>\mathrm{HC}$ \\
\hline \multicolumn{8}{|l|}{ Clinical parameters } \\
\hline HAMD total score & $10.73(7.55)$ & $21.83(5.26)$ & - & -7.55 & 85 & $0.000 * * *$ & $\mathrm{BD}<\mathrm{MDD}$ \\
\hline YMRS total score & $10.07(11.31)$ & - & - & - & - & - & - \\
\hline GAF & $54.11(13.63)$ & $53.10(10.05)$ & - & 0.18 & 73 & 0.73 & - \\
\hline Age first episode (years) & $23.85(10.00)$ & $27.21(11.20)$ & - & -1.41 & 78 & 0.16 & - \\
\hline Duration of illness (months) & $64.60(59.11)$ & $39.20(54.97)$ & - & 2.02 & 85 & $0.046 *$ & - \\
\hline Depressive episodes (n) & $2.24(1.46)$ & $1.77(1.07)$ & - & 1.50 & 70 & 0.14 & - \\
\hline Manic/hypomanic episodes (n) & $1.98(1.62)$ & - & - & - & - & - & - \\
\hline Mood stabilizers (n of users) & $30 / 52$ & $0 / 35$ & - & 30.82 & 1 & $0.000 * * *$ & $\mathrm{BD}>\mathrm{MDD}$ \\
\hline Antipsychotics (n of users) & $31 / 52$ & $3 / 35$ & - & 22.89 & 1 & $0.000 * \star \star$ & $\mathrm{BD}>\mathrm{MDD}$ \\
\hline
\end{tabular}

1.BD: bipolar disorder; MDD: major depressive disorder; HC: healthy controls; WAIS: Wechsler Adult Intelligence Scale Vocabulary Score (37 items); IQ, intelligence quotient; VIQ: verbal intelligence quotient; PIQ: performance intelligence quotient; TMT-A: Trail Making Test Parts A; TMT-B: Trail Making Test Parts B; DSST: digit symbol substitution test; C1q: Complement component 1q; C3: Complement component 3 ; C4: Complement component 4; Factor B: Complement factor B; Factor H: Complement factor B; HAMD: Hamilton depression rating scale (17 items); YMRS: Young Mania Rating Scale (11 items); GAF: Global Assessment of Functioning; n: number; SD: standard variance; M: male; F: female. For the comparison of complement factors, age, gender, education years and BMI were co-variated out.

2. ${ }^{*} p<0.05,{ }^{*} p<0.01,{ }^{* *} p<0.001$. Bold represents statistically significant results $(p<0.05)$ 


\begin{tabular}{|c|c|c|c|c|c|c|c|}
\hline & \multirow{2}{*}{$\begin{array}{l}\text { BD M (SD) } \\
(n=52)\end{array}$} & \multirow{2}{*}{$\begin{array}{l}\text { MDD M (SD) } \\
(n=35)\end{array}$} & \multirow{2}{*}{$\begin{array}{l}\mathrm{HCM}(S D) \\
(n=53)\end{array}$} & \multicolumn{4}{|c|}{ ANOVA } \\
\hline & & & & $F / x^{2}$ & df & P, 2-tail & Post hoc test \\
\hline Antidepressants (n of users) & $24 / 52$ & $18 / 35$ & - & 0.23 & 1 & 0.67 & - \\
\hline \multicolumn{8}{|c|}{$\begin{array}{l}\text { 1.BD: bipolar disorder; MDD: major depressive disorder; HC: healthy controls; WAIS: Wechsler Adult Intelligence Scale Vocabulary Score (37 } \\
\text { items); IQ, intelligence quotient; VIQ: verbal intelligence quotient; PIQ: performance intelligence quotient; TMT-A: Trail Making Test Parts A; } \\
\text { TMT-B: Trail Making Test Parts B; DSST: digit symbol substitution test; C1q: Complement component 1q; C3: Complement component } 3 \text {; } \\
\text { C4: Complement component 4; Factor B: Complement factor B; Factor H: Complement factor B; HAMD: Hamilton depression rating scale } \\
\text { (17 items); YMRS: Young Mania Rating Scale (11 items); GAF: Global Assessment of Functioning; n: number; SD: standard variance; M: } \\
\text { male; F: female. For the comparison of complement factors, age, gender, education years and BMI were co-variated out. }\end{array}$} \\
\hline$<0.05$ & Id repres & tically signi & sults ( $p$ & & & & \\
\hline
\end{tabular}

\section{Cognitive function}

All subject groups had similar estimated average IQ scores (Table 1), but ANCOVA revealed a significant main effect of group (BD, MDD, or HC) on immediate or delayed logical memory recall. Post-hoc Bonferroni correction also showed significantly lower immediate and delayed logical memory recall in BD patients than in HCs, whereas no difference was detected between MDD and BD patients or between MDD patients and HCs.

\section{Complement component levels}

The $\log _{10}$-transformed concentrations of all complement components were higher in the BD group than in HCs. The same trend was also observed in the MDD group, except for C3. Levels of C3, C4, and factor H were higher in patients with BD than in those with MDD, after covarying for age, sex, education level, and BMI. (Table 1). No other significant correlations were found between complement component levels and clinical parameters in either patient group (Table S1). C3b was excluded from the analysis, as it was not detected in more than half of the samples.

\section{GMV and CT changes in BD and MDD patients}

Voxel-based comparison of GMV in the mOFC, middle cingulum, and right precentral gyrus using age, sex, education years, and intracranial volume as covariates indicated significant differences among the three groups (Table 2 and Fig. 1a). Post-hoc analysis indicated common brain volume deficits in the mOFC and middle cingulum in the two patient groups (Table 2 and Figs. 1b-c), and MDD patients additionally showed lower GMV in the left precentral gyrus than HCs (Table 2 and Fig. 1c). 
Table 2

Significant difference of gray matter volume and cortical thickness between BD, MDD and HC group.

\begin{tabular}{|c|c|c|c|c|c|c|c|c|}
\hline \multirow[t]{2}{*}{ Model } & \multirow{2}{*}{$\begin{array}{l}\text { Cluster Size } \\
\text { (voxels) }\end{array}$} & \multirow{2}{*}{$\begin{array}{l}\mathrm{F} / \mathrm{t} \\
\text { value }\end{array}$} & \multirow{2}{*}{$\begin{array}{l}\text { Corrected } \\
\text { P }\end{array}$} & \multicolumn{3}{|c|}{ MNI Coordinates } & \multirow[t]{2}{*}{ BA } & \multirow{2}{*}{$\begin{array}{l}\text { Anatomical } \\
\text { Label }\end{array}$} \\
\hline & & & & $x$ & $Y$ & $\mathbf{Z}$ & & \\
\hline \multicolumn{9}{|l|}{ Gray matter volume } \\
\hline \multirow[t]{3}{*}{$\begin{array}{l}\text { Three group } \\
\text { comparison }\end{array}$} & 1234 & 18.52 & $0.000 * * *$ & $-8 / 5 / 2$ & $-26 /-18 /-12$ & $39 / 48 / 56$ & BA31/BA6/BA24 & $\begin{array}{l}\text { Middle } \\
\text { cingulum }\end{array}$ \\
\hline & 1582 & 13.91 & $0.000 * \star \star$ & $-12 /-2 /-3$ & $53 / 33 / 42$ & $-6 /-15 /-12$ & BA10/BA11 & $\begin{array}{l}\text { Frontal } \\
\text { medial } \\
\text { orbital } \\
\text { cortex }\end{array}$ \\
\hline & 598 & 12.56 & $0.016 *$ & $30 / 6 / 20$ & $-30 /-35 /-26$ & $56 / 72 / 65$ & BA4/BA6 & $\begin{array}{l}\text { Right } \\
\text { precentral } \\
\text { gyrus }\end{array}$ \\
\hline \multirow[t]{2}{*}{ Post-hoc: $\mathrm{BD}<\mathrm{HC}$} & 2040 & 5.84 & $0.000 * * \star$ & $-8 / 5 /-15$ & $-26 /-18 /-38$ & $38 / 48 / 38$ & BA31 & $\begin{array}{l}\text { Middle } \\
\text { cingulum }\end{array}$ \\
\hline & 2202 & 4.80 & $0.000 * \star \star$ & $-2 /-12 /-9$ & $33 / 53 / 47$ & $-15 /-8 /-12$ & BA10/BA11 & $\begin{array}{l}\text { Frontal } \\
\text { medial } \\
\text { orbital } \\
\text { cortex }\end{array}$ \\
\hline \multirow[t]{3}{*}{$\begin{array}{l}\text { Post-hoc: MDD < } \\
\text { HC }\end{array}$} & 1306 & 4.98 & $0.002 * \star$ & $30 / 6 / 20$ & $-30 /-35 /-24$ & $56 / 72 / 66$ & BA4/BA6 & $\begin{array}{l}\text { Right } \\
\text { precentral } \\
\text { gyrus }\end{array}$ \\
\hline & 656 & 4.66 & $0.027 *$ & $-6 / 8$ & $-24 /-17$ & $42 / 51$ & BA31 & $\begin{array}{l}\text { Middle } \\
\text { cingulum }\end{array}$ \\
\hline & 1190 & 4.59 & $0.002^{\star \star}$ & $-14 /-14 /-6$ & $50 / 41 / 44$ & $-8 /-5 /-11$ & BA10/BA11 & $\begin{array}{l}\text { Frontal } \\
\text { medial } \\
\text { orbital } \\
\text { cortex }\end{array}$ \\
\hline \multicolumn{9}{|l|}{ Cortical thickness } \\
\hline \multirow[t]{2}{*}{$\begin{array}{l}\text { Three group } \\
\text { comparison }\end{array}$} & 104 & 12.83 & $0.014 *$ & $-36 /-28$ & $-16 /-17$ & $67 / 70$ & BA6 & $\begin{array}{l}\text { Left } \\
\text { precentral } \\
\text { gyrus }\end{array}$ \\
\hline & 85 & 10.47 & $0.029 *$ & -8 & 33 & 40 & BA8 & $\begin{array}{l}\text { Left } \\
\text { superior } \\
\text { frontal } \\
\text { gyrus }\end{array}$ \\
\hline Post-hoc: $\mathrm{BD}<\mathrm{HC}$ & 115 & 4.29 & $0.018 *$ & -8 & 32 & 41 & BA8 & $\begin{array}{l}\text { Left } \\
\text { superior } \\
\text { frontal } \\
\text { gyrus }\end{array}$ \\
\hline $\begin{array}{l}\text { Post-hoc: MDD < } \\
\text { HC }\end{array}$ & 421 & 4.93 & $0.000 * * *$ & $-36 /-28 /-21$ & $-16 /-17 /-5$ & $67 / 70 / 62$ & BA6 & $\begin{array}{l}\text { Left } \\
\text { precentral } \\
\text { gyrus }\end{array}$ \\
\hline \multicolumn{9}{|c|}{ 1. BD: bipolar disorder; MDD: major depressive disorder; HC: healthy controls; BA: Brodmann area; MNI: Montreal Neurological Institute. } \\
\hline
\end{tabular}

Vertex-based comparisons of CT in the left precentral gyrus and left SFG revealed significant differences among the three groups after covarying for age, sex, and education level (Table 2 and Fig. 1d). CT of the left precentral gyrus was significantly smaller in the MDD group than in HCs, while CT in the left SFG was significantly smaller in the BD group than in HCs (Table 2 and Fig. 1d). However, no significant differences were observed in GMV or CT between BD and MDD patients after controlling for individual variations in intracranial volume (for GMV), age, sex, and education level.

\section{Correlation of complement component levels in plasma with whole-brain GMV or CT}


Whole-brain correlation analysis across all subjects after controlling for age, sex, education level, BMI, and TIV revealed a negative association between GMV in the mOFC and $\log _{10}$-transformed $\mathrm{C} 1 \mathrm{q}$, factor $\mathrm{H}$, and properdin (Table 3 and Fig. 2). However, whole-brain correlation analysis in each group did not reveal any significant correlation between GMV and plasma levels of complement components. We did not detect any significant results for CT among all subjects and in the individual groups after multiple correction analysis (Table S2).

Table 3

Whole brain-based negative correlation analysis of gray matter volume with complement component factors.

\begin{tabular}{|c|c|c|c|c|c|c|c|c|c|c|}
\hline \multirow[t]{2}{*}{ Samples } & \multirow{2}{*}{$\begin{array}{l}\text { Complement } \\
\text { Component }\end{array}$} & \multirow[t]{2}{*}{ Lateral } & \multirow[t]{2}{*}{ Cluster } & \multirow{2}{*}{$\begin{array}{l}\text { P } \\
\text { cluster }\end{array}$} & \multirow[t]{2}{*}{ BA } & \multirow{2}{*}{$\begin{array}{l}\text { Anatomical } \\
\text { label }\end{array}$} & \multirow[t]{2}{*}{$\mathrm{t}$} & \multicolumn{3}{|c|}{ MNI Peak coordinate } \\
\hline & & & & & & & & $x$ & $\mathbf{Y}$ & $\mathbf{Z}$ \\
\hline \multirow[t]{3}{*}{$\begin{array}{l}\text { All } \\
\text { subjects }\end{array}$} & $\log _{10} \mathrm{C1q}$ & Bilateral & 1343 & 0.001 ** & 11 & $\begin{array}{l}\text { Medial } \\
\text { orbital } \\
\text { frontal } \\
\text { cortex }\end{array}$ & $4.46 / 4.27 / 3.93$ & $-2 / 5 /-8$ & $32 / 41 / 43$ & $-21 /-18 /-15$ \\
\hline & $\log _{10} \mathrm{FH}$ & Bilateral & 903 & $0.008 * \star$ & 11 & $\begin{array}{l}\text { Medial } \\
\text { orbital } \\
\text { frontal } \\
\text { cortex }\end{array}$ & $4.32 / 4.18 / 3.59$ & $0 / 5 /-9$ & $33 / 42 / 54$ & $-23 /-18 /-14$ \\
\hline & $\begin{array}{l}\log _{10} \\
\text { Properdin }\end{array}$ & Bilateral & 1456 & 0.001 ** & $10 / 11$ & $\begin{array}{l}\text { Medial } \\
\text { orbital } \\
\text { frontal } \\
\text { cortex }\end{array}$ & $4.20 / 4.00 / 3.93$ & $0 / 0 /-6$ & $35 / 42 / 48$ & $-21 /-17 /-9$ \\
\hline
\end{tabular}

1. Age, sex, education years and body mass index were controlled. BA: Brodmann area, MNI: Montreal Neurological Institute.

2. Voxel-wise threshold was set at $p<0.0005$. Significance level was set at $p<0.05$, cluster wise family wise error corrected. ${ }^{\star} p<0.05$, $\star \star p<$ $0.01,{ }^{* *} p<0.001$. Bold represents statistically significant results. Bold represents statistically significant results.

\section{Correlation of cognitive function and altered brain structure}

Across all subjects, the GMV in the left mOFC positively correlated with immediate logical memory recall $(r=0.20, P=0.023)$, while the CT of the right precentral gyrus positively correlated with immediate logical memory recall $(r=0.18, P=0.047)$ and delayed logical memory recall $(r=$ $0.23, P=0.010$ ). However, these relationships were not significant after correction for multiple comparisons (Table S2). Therefore, we could not identify any specific correlation between disrupted brain structure and cognitive function.

\section{Discussion}

In the present study, we examined the association between complement components and brain structure deficits in BD and MDD patients, and found that the two disorders have common and distinct patterns of structural alterations. Compared to HCs, both patient groups showed reduced GMV in the middle cingulum and mOFC. In addition, MDD patients showed lower GMV in the right precentral gyrus and smaller CT in the left precentral gyrus than HCs, while the BD group showed smaller CT in the left superior frontal gyrus than HCs. Complement component levels were significantly higher in both patient groups than in $\mathrm{HCs}$, consistent with previous findings suggesting that both disorders are associated with hyperinflammatory responses $[50,51]$. In fact, the $\log _{10}$-transformed levels of $\mathrm{C} 3$, C4, and factor $\mathrm{H}$ were significantly higher in BD than in MDD patients, even after controlling for age, sex, years of education, and BMI. These results confirm our hypothesis that BD involves a more severe inflammatory response than MDD. Furthermore, we found that $\mathrm{C} 1 \mathrm{q}$, factor $\mathrm{H}$, and properdin were negatively associated with $\mathrm{GMV}$ in the bilateral mOFC across all subjects. To the best of our knowledge, this is the first HC-controlled study examining relationships between brain structure and complement components in mood disorders.

Our findings of shared structural alterations in the brains of BD and MDD patients are consistent with the results of a recent meta-analysis, where the volume of the ventromedial prefrontal cortex was found to be lower in both patient groups, suggesting a consistent volume deficit pattern in the two disorders [13]. A previous study also reported significantly smaller middle cingulum volume in MDD patients who did not respond to treatment than in $\mathrm{HCs}$ and in patients who did respond to treatment [52]. Although no similar reduction has been reported for BD patients, our results suggest that altered middle cingulate gyrus volume may underlie abnormal brain activation in both disorders. Our study also showed that MDD patients have significantly smaller GMV in the precentral and postcentral gyri as well as smaller CT in the left precentral gyrus than HCs. As both the precentral and postcentral gyri are key parts of the sensorimotor network [53], our results confirm the potential role of the precentral region in MDD pathogenesis [54].

MRI studies have implicated the SFG, the gyral-based representative of the dorsolateral prefrontal cortex [1], in self-awareness, complex cognitive behavior, executive function and emotional regulation [55]. In the present study, BD patients showed significantly smaller CT in the left SFG than HCs did. Our results are consistent with a previous whole-brain meta-analysis showing widespread cortical thinning in the SFG of 
BD patients, suggesting that structural deficits in the SFG may reflect the main emotional regulatory and processing symptoms of BD [56]. However, we did not find significant differences in GMV or CT between MDD and BD patients, similar to a recent study showing that brain structural data could not differentiate the two types of patients [13].

The BD group of the present study showed higher levels of all complement components than HCs. In addition, BD patients showed higher levels of $\mathrm{C} 3, \mathrm{C} 4$, and factor $\mathrm{H}$ than MDD patients. Our results are consistent with a previous report that BD patients have higher levels of proinflammatory cytokines than MDD patients [31], but our results should be verified in further studies of complement components in the two disorders. Interestingly, significantly increased levels of C3, C4, and factor B have also been detected in the serum plasma of patients with mania $[29,57]$. All these observations suggest that complement components may serve as effective biomarkers of mood disorders.

Factor $\mathrm{H}$ and properdin, which are key regulators of the alternative complement pathway [58,59], were also significantly increased in the $\mathrm{BD}$ group in our study. Although the roles of factor $\mathrm{H}$ and properdin in mood disorders remain unclear, dysregulation of the alternative pathway in neurological and neuropsychiatric disorders, including schizophrenia and epilepsy, has been reported [60, 61]. Consistent with these results, we found here that the levels of complement factors involved in the classical and alternative complement pathways were significantly increased in BD patients, suggesting that the complement system might be involved in disease neuropathology.

Levels of all complement components except C3 were significantly higher in MDD patients than in HCs in our study. Although our results are inconsistent with previous studies reporting high C3 levels in MDD patients [22], other studies have found high peripheral protein levels for C4, but not for C3, in patients with MDD [62]. Even allowing for differences in specific components, our results and the literature concur in supporting the idea that elevated levels of complement components may lead to hyperinflammatory responses in MDD.

Our results indicated a significant association of increased $\mathrm{C1q}$, factor $\mathrm{H}$, and properdin with GMV reduction in the mOFC of HCs and patients. The mOFC is a stress-sensitive brain area [63] involved in the regulation of emotions, cognition, and innate immunological responses to stress $[64,65]$. Decreased medial prefrontal cortex volume has also been widely reported as a neural abnormality in BD and MDD [64, 65]. Human and rodent studies have shown that in mood disorders, repeated environmental stress can activate innate immune receptors, such as Toll-like receptors, through exogenous or endogenous ligands, thereby evoking inflammation and attenuating neuronal response, while inducing dendritic atrophy and microglial activation in the medial prefrontal cortex [66]. Given also that C1q is the initiator molecule of the classical pathway, and factor $\mathrm{H}$ and properdin are key regulators of the alternative pathway [58,59], the present study suggests that both complement pathways might contribute to MPFC structural deficits in BD and MDD.

\section{Limitations}

Our study has some limitations, including its cross-sectional design. The single assessment of peripheral blood samples could not provide evidence of causality, leaving open the question of whether the observed alterations in complement levels and brain structure are a cause or consequence of BD and MDD [67]. Since C1q is associated with illness duration, longitudinal studies should be performed to explore the effect of illness duration on the expression of inflammatory markers. Moreover, the association between elevated complement components and mOFC structural changes needs to be carefully interpreted. In the present study, patients with severe physical illnesses, such as systemic lupus erythematosus, were excluded, and blood samples were collected from all subjects at the same time to avoid potential confounding due to variation in inflammation state. However, other factors affecting complement levels were not taken into account, such as concurrent immunological conditions (infections, atopy, autoimmune disorders) or sleep level [68], so future studies should address potential confounding from pre-existing inflammatory conditions. Furthermore, our BD patients did not show severe symptoms, although they showed increased levels of inflammatory markers, which may limit the generalizability of our findings to different BD subpopulations. All our BD patients had been receiving medications, so we were unable to assess the effects of treatment history on our results.

\section{Conclusion And Outlook}

To the best of our knowledge, our study is the first to compare structural neuroimaging analysis with complement factor levels in BD, MDD, and HCs. Consistent with previous brain structural findings, we found that BD and MDD patients have common and distinct patterns of GMV and CT deficits, and that BD involves more severe inflammatory responses than MDD. We expect that this study will help improve our understanding of the association between peripheral inflammation and altered brain structure in bipolar disorders.

\section{Abbreviations}

BD

Bipolar disorders

MDD

Major depressive disorders 
GMV

Gray matter volume

CT

Cortical thickness

VBM

Voxel-based morphometry

SBM

Surface-based morphometry

MOFC

Medial orbital frontal cortex

ACC

Anterior cingulate cortex

SFG

Superior frontal gyrus

IL-6

Interleukin 6

BMI

Body mass index

$\mathrm{HC}$

Healthy controls

IQ

Intelligence quotient

MRI

Magnetic resonance imaging

TIV

Total intracranial volume

FWHM

Full-width half-maximum

ANCOVA

Analysis of covariance

\section{Declarations}

\section{Availability of data and materials}

All data used in the current study are available from the corresponding author on reasonable request.

\section{Acknowledgments}

The authors would like to thank all their coworkers at West China Hospital, the State Key Laboratory of Biotherapy in West China Hospital, Suzhou Psychiatry Hospital, and the Affiliated Mental Health Centre \& Hangzhou Seventh People's Hospital for their contributions to this research study.

\section{Funding}

This work was supported by the National Natural Science Foundation of China Key Project (81630030 and 81920108018 to T.L.), National Natural Science Foundation of China (81871054 and 81501159 to P.N., 8210051503 to H.Y.), Special Foundation for Brain Research from Science and Technology Program of Guangdong (2018B030334001), 2021 Project for Hangzhou Medical Disciplines of Excellence \& Key Project for Hangzhou Medical Disciplines, 1.3.5 Project for Disciplines of Excellence at West China Hospital of Sichuan University (ZY2016103, ZY2016203, and ZYGD20004 to T.L.), and Introductory Project of the Suzhou Clinical Expert Team (SZYJTD201715 to X.D. and T.L.).

\section{Author information}

Hua Yu and Peiyan Ni contributed equally to this work

\section{Affiliations}

The Psychiatric Laboratory, State Key Laboratory of Biotherapy, West China Hospital, Sichuan University, Chengdu, Sichuan, P R China 
Hua Yu, Peiyan Ni, Yang Tian, Liansheng Zhao, Mingli Li, Xiaojing Li, Wei Wei, Jinxue Wei, Qiang Wang, Wanjun Guo, Xiaohong Ma, Jeremy Coid, Tao Li.

\section{Mental Health Center, West China Hospital of Sichuan University, Chengdu, China}

Hua Yu, Peiyan Ni, Yang Tian, Liansheng Zhao, Mingli Li, Xiaojing Li, Wei Wei, Jinxue Wei, Qiang Wang, Wanjun Guo, Xiaohong Ma, Jeremy Coid, Tao Li.

\section{Suzhou Psychiatry Hospital, Affiliated Guangji Hospital of Soochow University, Suzhou, 215137, Jiangsu, China}

Xiangdong Du

\section{Affiliated Mental Health Centre \& Hangzhou Seventh People's Hospital, Zhejiang University School of Medicine, Hangzhou, 310013, Zhejiang, China}

Wei Deng, Tao Li

\section{Contributions}

H.Y., P.N., J.C., and T.L. developed the study, had full access to all data, and take responsibility for data integrity and accuracy. H.Y., P.N., Q.W., and W.G. drafted the manuscript. W.W., J.W., X.D., W.D., and X.M. collected the data, and Y.T., L.Z., M.L., and X.L. performed all data analyses. All authors agree to be accountable for all aspects of the work, ensuring that questions related to the accuracy or integrity of the data and results are appropriately investigated and resolved. All authors critically revised and approved the final version of the manuscript.

\section{Corresponding author}

Correspondence to Tao Li.

\section{Ethics declarations}

\section{Ethics approval and consent to participate}

The study was approved by the Ethics Committee of West China Hospital, Sichuan University, and it complied with the principles of the Declaration of Helsinki. All participants received a complete description of the study and provided written informed consent. A copy of the written consent is available for review by the Editor of this journal.

\section{Consent for publication}

Not applicable.

\section{Competing interests}

The authors declare no conflicts of interest.

\section{Publisher's Note}

Springer Nature remains neutral with regard to jurisdictional claims in published maps and institutional affiliations.

\section{References}

1. Niu M, Wang Y, Jia Y, Wang J, Zhong S, Lin J, Sun Y, Zhao L, Liu X, Huang L, Huang R. Common and Specific Abnormalities in Cortical Thickness in Patients with Major Depressive and Bipolar Disorders. EBioMedicine. 2017;16:162-71.

2. Hasin DS, Goodwin RD, Stinson FS, Grant BF. Epidemiology of major depressive disorder: results from the National Epidemiologic Survey on Alcoholism and Related Conditions. Arch Gen Psychiatry. 2005;62:1097-106.

3. Merikangas KR, Akiskal HS, Angst J, Greenberg PE, Hirschfeld RMA, Petukhova M, Kessler RC. Lifetime and 12-Month Prevalence of Bipolar Spectrum Disorder in the National Comorbidity Survey Replication. Arch Gen Psychiatry. 2007;64:543-52.

4. Gitlin M. Treatment-resistant bipolar disorder. Mol Psychiatry. 2006;11:227-40.

5. MacDonald K, Krishnan A, Cervenka E, Hu G, Guadagno E, Trakadis Y. Biomarkers for major depressive and bipolar disorders using metabolomics: A systematic review. American Journal of Medical Genetics Part B: Neuropsychiatric Genetics. 2019;180:122-37. 
6. Han K-M, De Berardis D, Fornaro M, Kim Y-K. Differentiating between bipolar and unipolar depression in functional and structural MRI studies. Prog Neuropsychopharmacol Biol Psychiatry. 2019;91:20-7.

7. Rosenblat JD, Kakar R, Berk M, Kessing LV, Vinberg M, Baune BT, Mansur RB, Brietzke E, Goldstein BI, Mclntyre RS. Anti-inflammatory agents in the treatment of bipolar depression: a systematic review and meta-analysis. Bipolar Disord. 2016;18:89-101.

8. Felger JC. The Role of Dopamine in Inflammation-Associated Depression: Mechanisms and Therapeutic Implications. Curr Top Behav Neurosci. 2017;31:199-219.

9. Opel N, Goltermann J, Hermesdorf M, Berger K, Baune BT, Dannlowski U. Cross-Disorder Analysis of Brain Structural Abnormalities in Six Major Psychiatric Disorders: A Secondary Analysis of Mega- and Meta-analytical Findings From the ENIGMA Consortium. Biol Psychiatry. 2020;88:678-86.

10. Lan MJ, Chhetry BT, Oquendo MA, Sublette ME, Sullivan G, Mann JJ, Parsey RV. Cortical thickness differences between bipolar depression and major depressive disorder. Bipolar Disord. 2014;16:378-88.

11. Kong L, Herold CJ, Zöllner F, Salat DH, Lässer MM, Schmid LA, Fellhauer I, Thomann PA, Essig M, Schad LR, et al. Comparison of grey matter volume and thickness for analysing cortical changes in chronic schizophrenia: A matter of surface area, grey/white matter intensity contrast, and curvature. Psychiatry Research: Neuroimaging. 2015;231:176-83.

12. Phillips ML, Swartz HA. A Critical Appraisal of Neuroimaging Studies of Bipolar Disorder: Toward a New Conceptualization of Underlying Neural Circuitry and a Road Map for Future Research. Am J Psychiatry. 2014;171:829-43.

13. Wise T, Radua J, Via E, Cardoner N, Abe O, Adams TM, Amico F, Cheng Y, Cole JH de Azevedo Marques Périco C, et al: Common and distinct patterns of grey-matter volume alteration in major depression and bipolar disorder: evidence from voxel-based meta-analysis. Mol Psychiatry 2017, 22:1455-1463.

14. Yu H, Meng Y-j, Li X-j, Zhang C, Liang S, Li M-I, Li Z, Guo W, Wang Q, Deng W, et al. Common and distinct patterns of grey matter alterations in borderline personality disorder and bipolar disorder: voxel-based meta-analysis. The British Journal of Psychiatry. 2019;215:395-403.

15. Chen L, Wang Y, Niu C, Zhong S, Hu H, Chen P, Zhang S, Chen G, Deng F, Lai S, et al. Common and distinct abnormal frontal-limbic system structural and functional patterns in patients with major depression and bipolar disorder. Neuroimage Clin. 2018;20:42-50.

16. Fung G, Deng Y, Zhao Q, Li Z, Qu M, Li K, Zeng YW, Jin Z, Ma YT, Yu X, et al. Distinguishing bipolar and major depressive disorders by brain structural morphometry: a pilot study. BMC Psychiatry. 2015;15:298.

17. Smoller JW. The Genetics of Stress-Related Disorders: PTSD, Depression, and Anxiety Disorders. Neuropsychopharmacology. 2016;41:297-319.

18. Benedetti F, Aggio V, Pratesi ML, Greco G, Furlan R. Neuroinflammation in Bipolar Depression. Front Psychiatry. $2020 ; 11: 71$.

19. Berk M, Kapczinski F, Andreazza AC, Dean OM, Giorlando F, Maes M, Yücel M, Gama CS, Dodd S, Dean B, et al. Pathways underlying neuroprogression in bipolar disorder: focus on inflammation, oxidative stress and neurotrophic factors. Neurosci Biobehav Rev. 2011;35:804-17.

20. Green C, Shen X, Stevenson AJ, Conole ELS, Harris MA, Barbu MC, Hawkins EL, Adams MJ, Hillary RF, Lawrie SM, et al: Structural brain correlates of serum and epigenetic markers of inflammation in major depressive disorder. Brain Behav Immun 2020.

21. Woo JJ, Pouget JG, Zai CC, Kennedy JL. The complement system in schizophrenia: where are we now and what's next? Mol Psychiatry. 2020;25:114-30.

22. Pillai A, Bruno D, Nierenberg J, Pandya C, Feng T, Reichert C, Ramos-Cejudo J, Osorio R, Zetterberg H, Blennow K, Pomara N. Complement component 3 levels in the cerebrospinal fluid of cognitively intact elderly individuals with major depressive disorder. Biomarkers in neuropsychiatry. 2019;1:100007.

23. Reid KBM. Complement Component C1q: Historical Perspective of a Functionally Versatile, and Structurally Unusual, Serum Protein. Front Immunol. 2018;9:764.

24. Kopczynska M, Zelek W, Touchard S, Gaughran F, Di Forti M, Mondelli V, Murray R, O'Donovan MC, Morgan BP. Complement system biomarkers in first episode psychosis. Schizophr Res. 2019;204:16-22.

25. Yao Q, Li Y. Increased serum levels of complement C1q in major depressive disorder. J Psychosom Res. 2020;133:110105.

26. Crider A, Feng T, Pandya CD, Davis T, Nair A, Ahmed AO, Baban B, Turecki G, Pillai A. Complement component 3a receptor deficiency attenuates chronic stress-induced monocyte infiltration and depressive-like behavior. Brain Behav Immun. 2018;70:246-56.

27. Shin C, Ham BJ, Ko YH, Pae CU, Park MH, Steffens DC, Patkar AA, Han C. Increased plasma complement factor H is associated with geriatric depression. Int Psychogeriatr. 2019;31:101-8.

28. Yang X, Tao H, Xiao L, Li C, Tang Y, Liu Y. Increased Serum C3 and Decreased UA in Patients of Bipolar Disorder in Chinese Han Population. Front Psychiatry. 2018;9:381.

29. Reginia A, Kucharska-Mazur J, Jabłoński M, Budkowska M, Dołðgowska B, Sagan L, Misiak B, Ratajczak MZ, Rybakowski JK, Samochowiec J. Assessment of Complement Cascade Components in Patients With Bipolar Disorder. Frontiers in psychiatry. 2018;9:614-

Page $13 / 17$ 
4.

30. Chang HH, Wang TY, Lee IH, Lee SY, Chen KC, Huang SY, Yang YK, Lu RB, Chen PS. C-reactive protein: A differential biomarker for major depressive disorder and bipolar II disorder. World J Biol Psychiatry. 2017;18:63-70.

31. Bai YM, Chen MH, Hsu JW, Huang KL, Tu PC, Chang WC, Su TP, Li CT, Lin WC, Tsai SJ. A comparison study of metabolic profiles, immunity, and brain gray matter volumes between patients with bipolar disorder and depressive disorder. J Neuroinflammation. 2020;17:42.

32. Harrison NA. Brain Structures Implicated in Inflammation-Associated Depression. Curr Top Behav Neurosci. 2017;31:221-48.

33. Meier TB, Drevets WC, Wurfel BE, Ford BN, Morris HM, Victor TA, Bodurka J, Teague TK, Dantzer R, Savitz J. Relationship between neurotoxic kynurenine metabolites and reductions in right medial prefrontal cortical thickness in major depressive disorder. Brain Behav Immun. 2016;53:39-48.

34. Maletic V, Raison C. Integrated Neurobiology of Bipolar Disorder. Front Psychiatry. 2014;5:98.

35. Sekar A, Bialas AR, de Rivera H, Davis A, Hammond TR, Kamitaki N, Tooley K, Presumey J, Baum M, Van Doren V, et al. Schizophrenia risk from complex variation of complement component 4. Nature. 2016;530:177-83.

36. Wu T, Dejanovic B, Gandham VD, Gogineni A, Edmonds R, Schauer S, Srinivasan K, Huntley MA, Wang Y, Wang T-M, et al. Complement C3 Is Activated in Human AD Brain and Is Required for Neurodegeneration in Mouse Models of Amyloidosis and Tauopathy. Cell Reports. 2019;28:2111-23.e2116.

37. Guglielmo R, Miskowiak KW, Hasler G. Evaluating endophenotypes for bipolar disorder. International Journal of Bipolar Disorders. 2021;9:17.

38. Hasler G, Drevets WC, Manji HK, Charney DS. Discovering Endophenotypes for Major Depression. Neuropsychopharmacology. 2004;29:1765-81.

39. Felger JC, Li Z, Haroon E, Woolwine BJ, Jung MY, Hu X, Miller AH. Inflammation is associated with decreased functional connectivity within corticostriatal reward circuitry in depression. Mol Psychiatry. 2016;21:1358-65.

40. First MB. Structured Clinical Interview for DSM-IV Axis I Disorders. Biometrics Research Department, 1997.

41. Young RC, Biggs JT, Ziegler VE, Meyer DA. A Rating Scale for Mania: Reliability, Validity and Sensitivity. Br J Psychiatry. 1978;133:429-35.

42. Hamilton M. A rating scale for depression. J Neurol Neurosurg Psychiatry. 1960;23:56-62.

43. First M, Spitzer RL, Gibbon ML, Williams J: Structured clinical interview for DSM-IV-TR Axis I Disorders, Research Version, Non-patient Edition. In; 2002.

44. Wechsler D. WAIS-R Manual: Wechsler Adult Intelligence Scale-revised. Psychological Corporation; 1981.

45. Abikoff H, Alvir J, Hong G, Sukoff R, Orazio J, Solomon S, Saravay S. Logical memory subtest of the Wechsler Memory Scale: age and education norms and alternate-form reliability of two scoring systems. J Clin Exp Neuropsychol. 1987;9:435-48.

46. Tombaugh TN. Trail Making Test A and B: normative data stratified by age and education. Arch Clin Neuropsychol. 2004;19:203-14.

47. Gaser C, Dahnke R. CAT-A Computational Anatomy Toolbox for the Analysis of Structural MRI Data. In.; 2016.

48. Zhang J, Wu Y-L, Su J, Yao Q, Wang M, Li G-F, Zhao R, Shi Y-H, Zhao Y, Zhang Q, et al. Assessment of gray and white matter structural alterations in migraineurs without aura. The Journal of Headache Pain. 2017;18:74.

49. Tseng HH, Chang HH, Wei SY, Lu TH, Hsieh YT, Yang YK, Chen PS. Peripheral inflammation is associated with dysfunctional corticostriatal circuitry and executive dysfunction in bipolar disorder patients. Brain Behav Immun. 2021;91:695-702.

50. Akcan U, Karabulut S, İsmail Küçükali C, Çakır S, Tüzün E. Bipolar disorder patients display reduced serum complement levels and elevated peripheral blood complement expression levels. Acta Neuropsychiatr. 2018;30:70-8.

51. Wei J, Liu Y, Zhao L, Yang X, Ni P, Wang Y, Li T, Ma X. Plasma complement component 4 increases in patients with major depressive disorder. Neuropsychiatr Dis Treat. 2018;14:37-41.

52. Batail JM, Coloigner J, Soulas M, Robert G, Barillot C, Drapier D. Structural abnormalities associated with poor outcome of a major depressive episode: The role of thalamus. Psychiatry Res Neuroimaging. 2020;305:111158.

53. Chenji S, Jha S, Lee D, Brown M, Seres P, Mah D, Kalra S. Investigating Default Mode and Sensorimotor Network Connectivity in Amyotrophic Lateral Sclerosis. PloS one. 2016;11:e0157443-3.

54. Maggioni E, Delvecchio G, Grottaroli M, Garzitto M, Piccin S, Bonivento C, Maieron M, D'Agostini S, Perna G, Balestrieri M, Brambilla P. Common and different neural markers in major depression and anxiety disorders: A pilot structural magnetic resonance imaging study. Psychiatry Research: Neuroimaging. 2019;290:42-50.

55. Yang Y, Liu S, Jiang X, Yu H, Ding S, Lu Y, Li W, Zhang H, Liu B, Cui Y, et al. Common and Specific Functional Activity Features in Schizophrenia, Major Depressive Disorder, and Bipolar Disorder. Front Psychiatry. 2019;10:52.

56. Hanford LC, Nazarov A, Hall GB, Sassi RB. Cortical thickness in bipolar disorder: a systematic review. Bipolar Disord. 2016;18:4-18. 
57. Wadee AA, Kuschke RH, Wood LA, Berk M, Ichim L, Maes M. Serological observations in patients suffering from acute manic episodes. Hum Psychopharmacol. 2002;17:175-9.

58. Noris M, Remuzzi G. Overview of Complement Activation and Regulation. Semin Nephrol. 2013;33:479-92.

59. Chen JY, Cortes C, Ferreira VP. Properdin: A multifaceted molecule involved in inflammation and diseases. Mol Immunol. $2018 ; 102: 58-72$.

60. Zhang C, Lv Q, Fan W, Tang W, Yi Z. Influence of CFH gene on symptom severity of schizophrenia. Neuropsychiatr Dis Treat. 2017;13:697706.

61. Kopczynska M, Zelek WM, Vespa S, Touchard S, Wardle M, Loveless S, Thomas RH, Hamandi K, Morgan BP. Complement system biomarkers in epilepsy. Seizure. 2018;60:1-7.

62. Berk M, Wadee AA, Kuschke RH, O'Neill-Kerr A. Acute phase proteins in major depression. J Psychosom Res. 1997;43:529-34.

63. McEwen BS, Morrison JH. The brain on stress: vulnerability and plasticity of the prefrontal cortex over the life course. Neuron. 2013;79:1629.

64. Savitz JB, Price JL, Drevets WC. Neuropathological and neuromorphometric abnormalities in bipolar disorder: View from the medial prefrontal cortical network. Neuroscience Biobehavioral Reviews. 2014;42:132-47.

65. Belleau EL, Treadway MT, Pizzagalli DA. The Impact of Stress and Major Depressive Disorder on Hippocampal and Medial Prefrontal Cortex Morphology. Biol Psychiatry. 2019;85:443-53.

66. Nie X, Kitaoka S, Tanaka K, Segi-Nishida E, Imoto Y, Ogawa A, Nakano F, Tomohiro A, Nakayama K, Taniguchi M, et al. The Innate Immune Receptors TLR2/4 Mediate Repeated Social Defeat Stress-Induced Social Avoidance through Prefrontal Microglial Activation. Neuron. 2018;99:464-79.e467.

67. Miklowitz DJ, Portnoff LC, Armstrong CC, Keenan-Miller D, Breen EC, Muscatell KA, Eisenberger NI, Irwin MR. Inflammatory cytokines and nuclear factor-kappa B activation in adolescents with bipolar and major depressive disorders. Psychiatry Res. 2016;241:315-22.

68. Nilsson B, Ekdahl KN. Complement diagnostics: concepts, indications, and practical guidelines. Clin Dev Immunol. 2012;2012:962702-2.

\section{Figures}




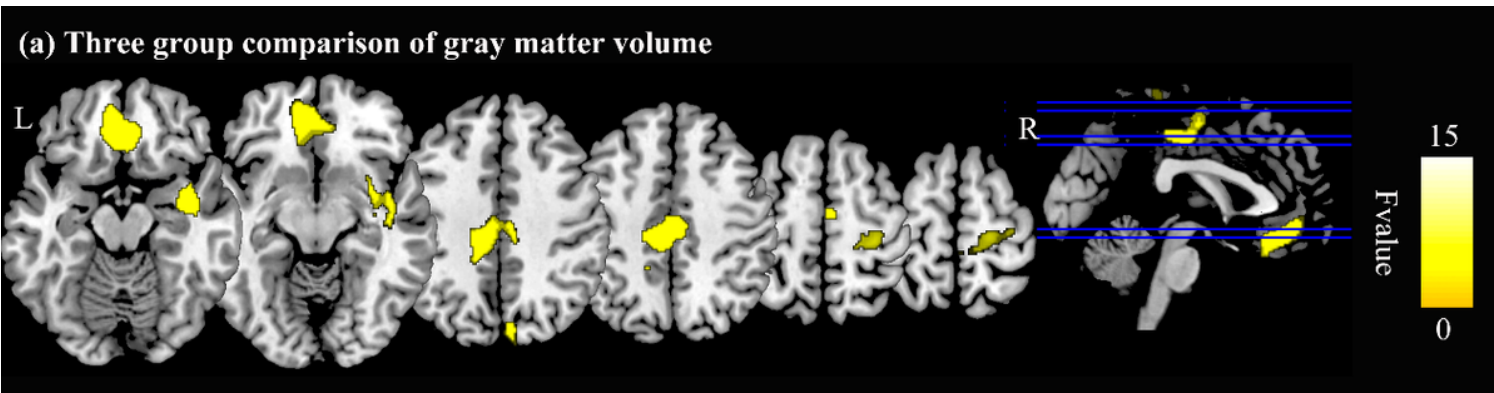

(b) Post-hoc analysis of gray matter volume: $\mathrm{BD}<\mathrm{HC}$

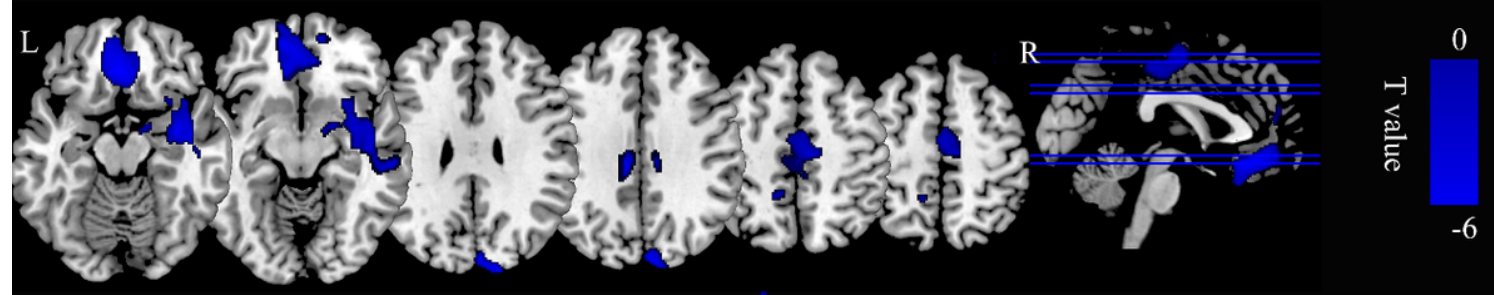

(c) Post-hoc analysis of gray matter volume: MDD $<$ HC

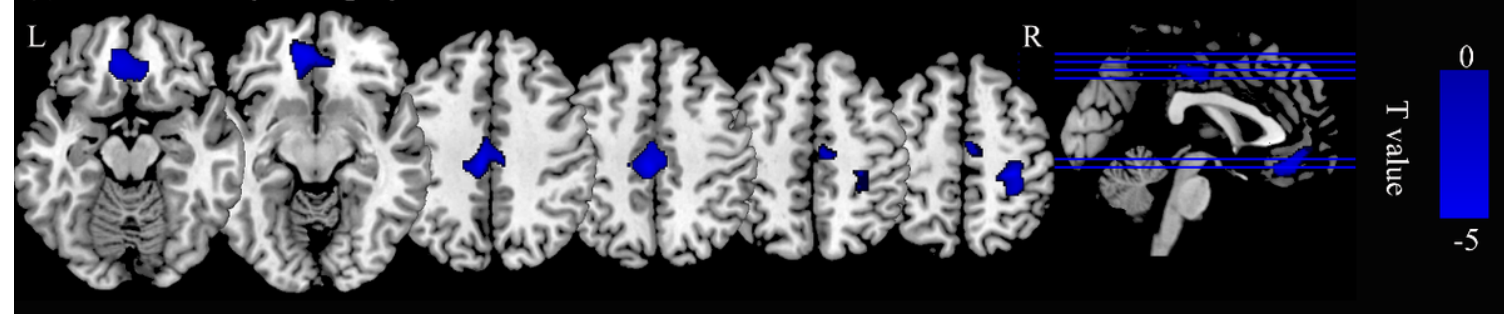

(d) Group comparison of cortical thickness

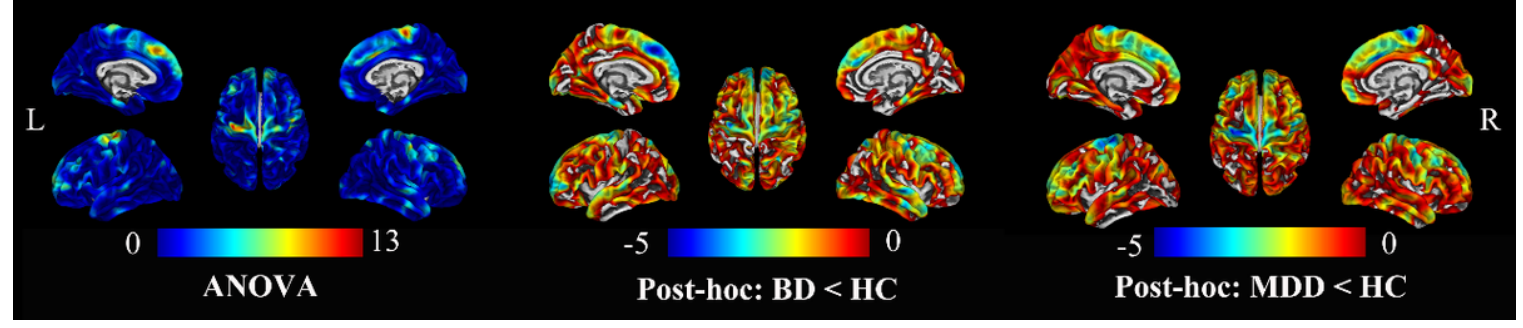

Figure 1

Gray matter volume and cortical thickness differences between participants with bipolar disorder and major depressive disorder, compared to healthy controls. (a). Three group comparisons: The gray matter volume of the middle cingulum, medial orbital frontal cortex, right superior temporal gyrus, right pre/post central gyrus and right occipital gyrus differed significantly among the three groups (color in yellow); (b). Compared with controls, bipolar disorder patients showed decreased gray matter volume in middle cingulum, right superior temporal gyrus, medial frontal orbital cortex and right superior occipital cortex (color in blue); (c). Compared with controls, the major depressive disorder patients showed decreased gray matter volume in right pre/postcentral gyrus, middle cingulum and medial orbital frontal cortex (color in blue); (d). The figure in the left showed three group comparison results of the cortical thickness, including bilateral precentral gyrus and left superior frontal gyrus (color in red); the figure in the middle showed that cortical thickness in the left superior frontal gyrus was decreased in bipolar patients group compared with controls (cold color); the figure in the right showed that compared with controls, major depressive disorder patients showed decreased cortical thickness in bilateral precentral gyrus (cold color). (All the results were co-variated out for age, gender and education years, and the total intracranial volume was additionally co-variated out for gray matter volume analysis, voxel threshold significance was set at $p<0.0005$, cluster level was set at $p<0.05$, family wise error corrected). BD: bipolar disorder, MDD: major depressive disorder, HC: healthy control. 


\section{a. The association of serum $\mathrm{Clq}$ level with gray matter volume of mOFC across all the subjects.}

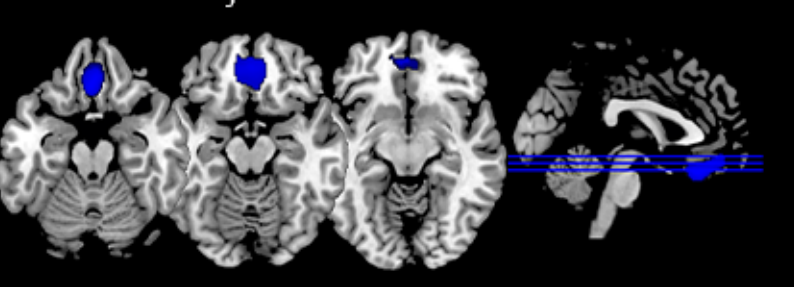

b. The association of serum factor $\mathrm{H}$ level with gray matter volume of mOFC across all the subjects.

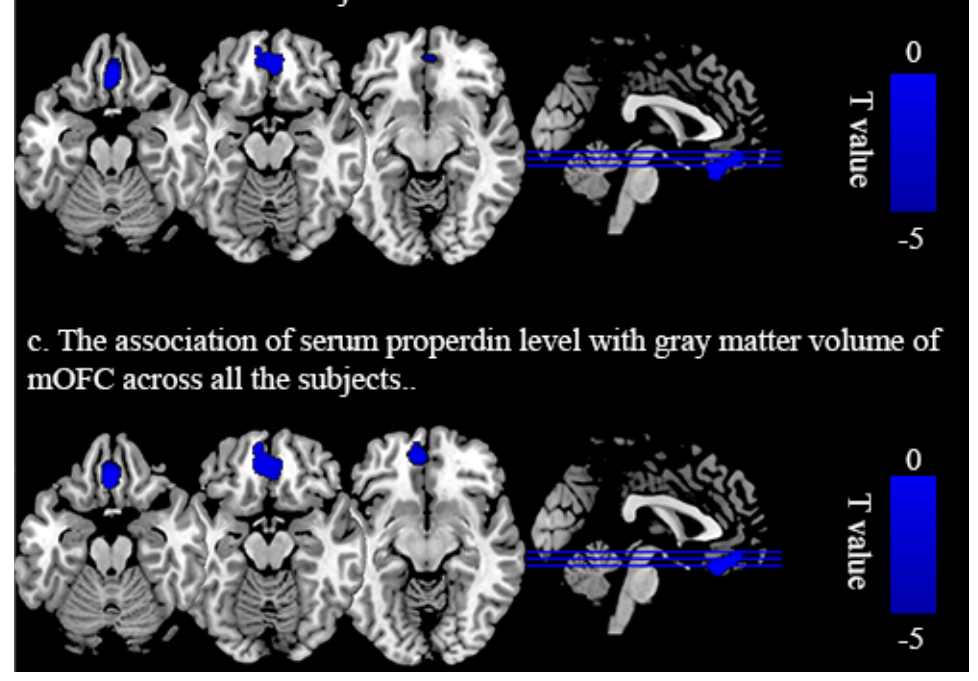

\section{Figure 2}

(a) Figure shows significant negative correlation between log10-transformed C1q and volume of medial orbital prefrontal cortex (cold color) across all the subjects. (b) Figure shows significant negative correlation of the log 10 -transformed factor $\mathrm{H}$ and gray matter volume in medial orbital prefrontal cortex (cold color) across all the subjects. (c). Figure shows significant negative correlation of the log10-transformed properdin and gray matter volume in medial orbital prefrontal cortex (cold color) across all the subjects. For the correlation analysis, age, gender, education years, total intracranial volume and body mass index were set as the covariates. Significance was set at a threshold with a peak-level uncorrected $p<0.0005$, with a family-wise error rate (FWE)-corrected cluster level of $p<0.05)$. MOFC: medial orbital frontal cortex.

\section{Supplementary Files}

This is a list of supplementary files associated with this preprint. Click to download.

- Supplementarymaterials.docx 